\title{
Covid 19 vaccines review
}

BY

Mohamed Ashraf Mohamed, M.A.M.,

Pharmaceutics department, Egyptian Russian University, ERU

Doi:10.21608/asajs.2021.143517

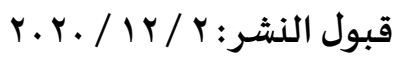

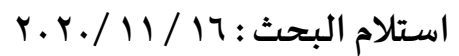

\section{Introduction}

Vaccine is the magic solution for saving the life in humans and animals. The mechanisms of different prepared vaccines immunity against different pathogens are illustrated in figure 1. The different vaccine platforms have their advantages and disadvantages. Live-attenuated platforms offer higher immunogenicity in spite of pose safety concerns due to retained pathogenicity of pathogens. The killed vaccines elicit moderate levels of immunogenicity and higher levels of safety compared to the live attenuated vaccine platforms ${ }^{[1] .}$

To combat Covid 19 vaccine, the behavioral and therapeutic strategies are essential but they must be accompanied with the long-term goal of a preventative vaccine virus. In the case of COVID-19, the pathogen is the virus SARS-CoV-2, a vaccine can use. A virus that has been modified to be safe or a molecule that resembles a part of the virus. Once antibodies are eliminated, if the vaccinated person is exposed later to the virus, their body will produce those antibodies again, increasing their chances of fighting off infection .Today, there are more than 150 COVID-19 vaccines in the race to mollify the virus. Researchers develop a vaccine candidate similar to that target that will induce production of antibodies effective against the virus. The vaccine candidate is then moved through phases of development, assessment, and approvals (figure. 2) ${ }^{[2]}$. 


\section{Doi:10.21608/asajs.2021.143517 Mohamed Ashraf Mohamed}

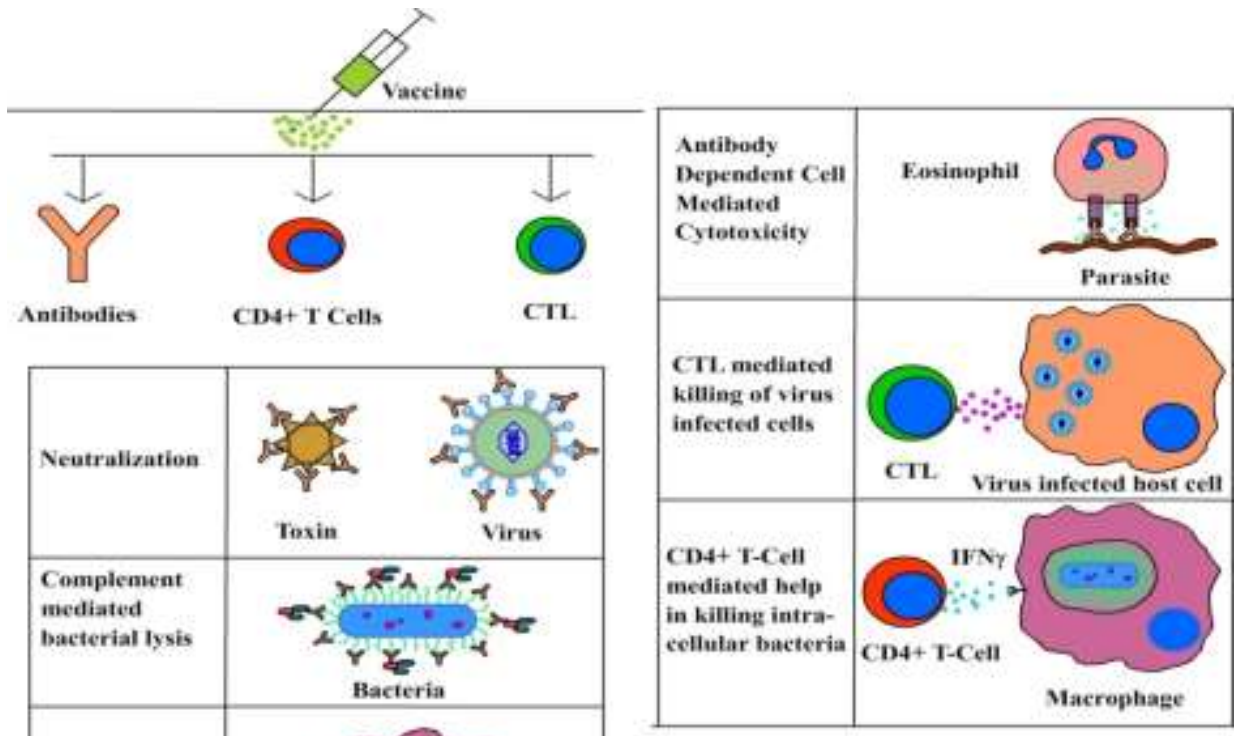

Opsonization

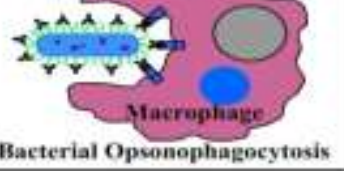

Figure 1. (Immunity response for administration of different prepared vaccines) 


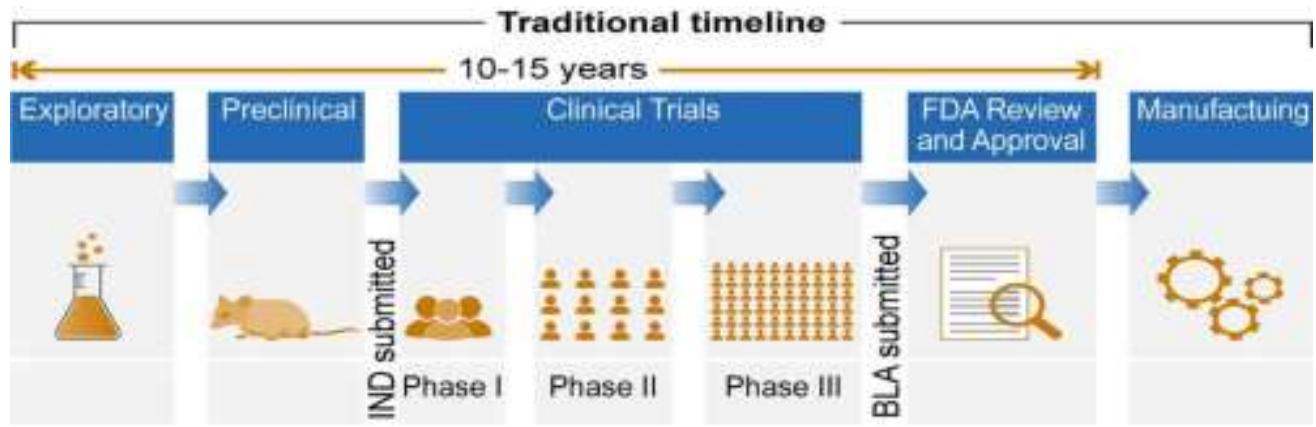

Potential accelerated timeline with overlapping phases and EUA

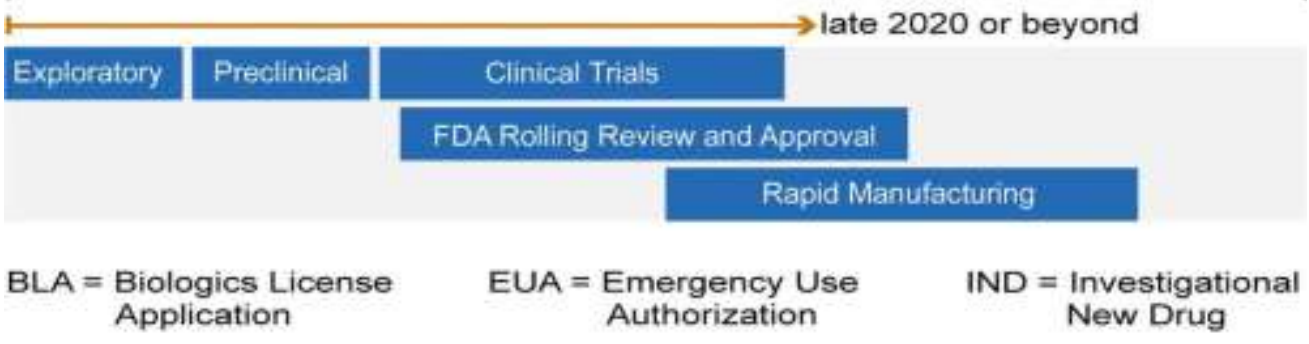

Source: GAO analysis of GAO-20-215SP, FDA, HHS, and Pharmaceutical Research and Manufacturers of America (PhRMA) documentation, | GAO-20-583SP

Figure (2). The vaccine development process almost takes 10 to 15 years under routine timeline. Several regulatory pathways, e.g. Emergency Use Authorization, can be applied to produce fast COVID-19 market vaccine

As of May 15, 2020, there are more than 110 COVID-19 vaccines in development globally; of those, at least three are being developed in the United States with federal funding. These three use different mechanisms to prompt the body to produce antibodies (fig. 3). Consecutive testing of several vaccine mechanisms able to improve the chances for effective development of a successful vaccine faster. One to three these vaccines have already reached Phase III clinical trials in less than a year; an unprecedented time ${ }^{[3]}$. 


\section{Doi:10.21608/asajs.2021.143517 Mohamed Ashraf Mohamed}

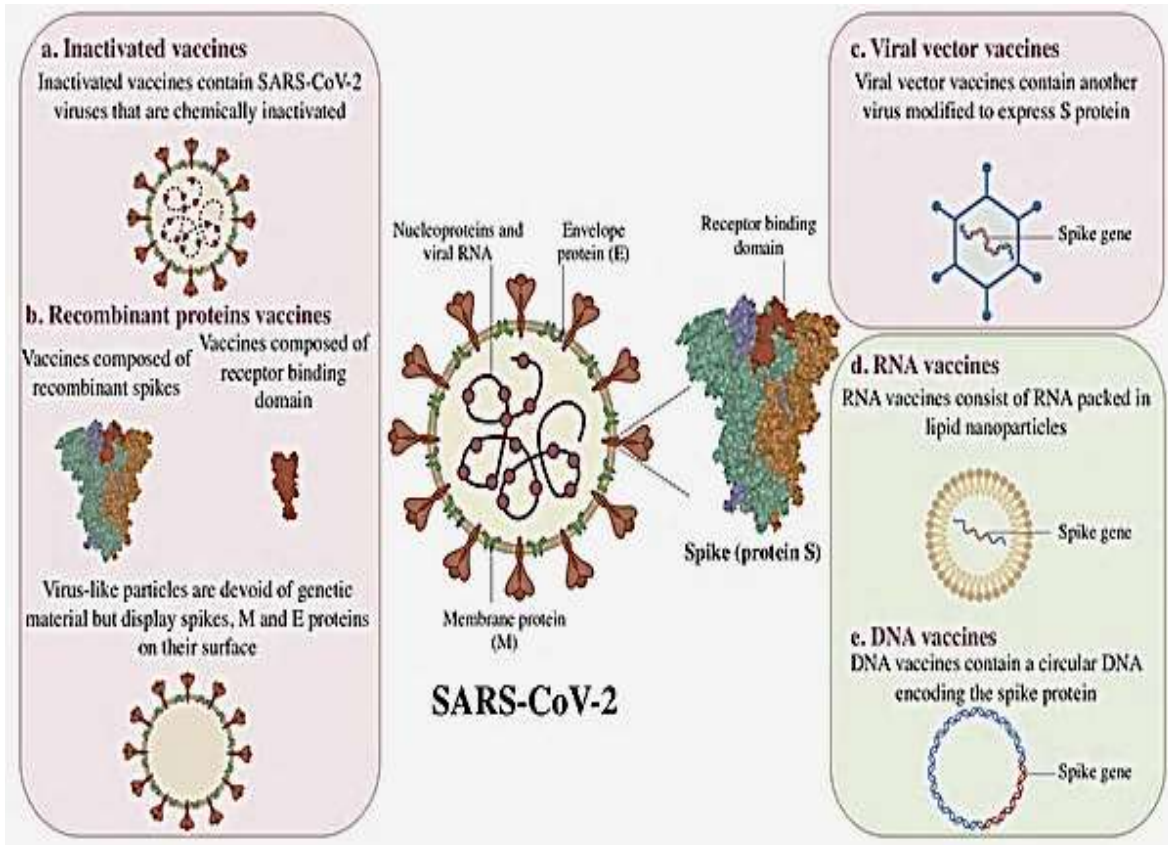

Figure (3) Vaccine candidates for production of covid19

\section{vaccines}

\section{Trials of vaccine candidates}

\section{Inactivated vaccines}

Since the 1880 s, inactivated vaccines have been used. The viruses in these vaccines had inactivated by chemical treatment, as with SARS-CoV-2 candidate vaccines, or by physical treatment. B-propiolactone inactivated aluminum-adjuvanted whole-virion SARS-CoV-2 vaccine developed by Wuhan Institute of Biological Products ${ }^{[4]}$ The immune system in these cases encounters the virus in its entirety. It can mount a defense when it detects the viral spike protein (spicule or S-protein), envelope and nucleoprotein. Currently, seven inactivated vaccine candidates are being tested in humans. Of these, three are in Phase 3 clinical trials. Unlike Phase 1 and Phase 2, which are used to evaluate a vaccine's tolerability, safety and ability to 
induce an immune response, a Phase 3 clinical trial allows scientists to test its efficacy ${ }^{[5]}$

Once the sequencing of SARS-CoV-2 gnome becomes available, several nucleic acid based vaccine candidates start proposing for preparation depends on S protein coding sequence. The different mechanisms developed by vaccine candidates are illustrated in figure (4).

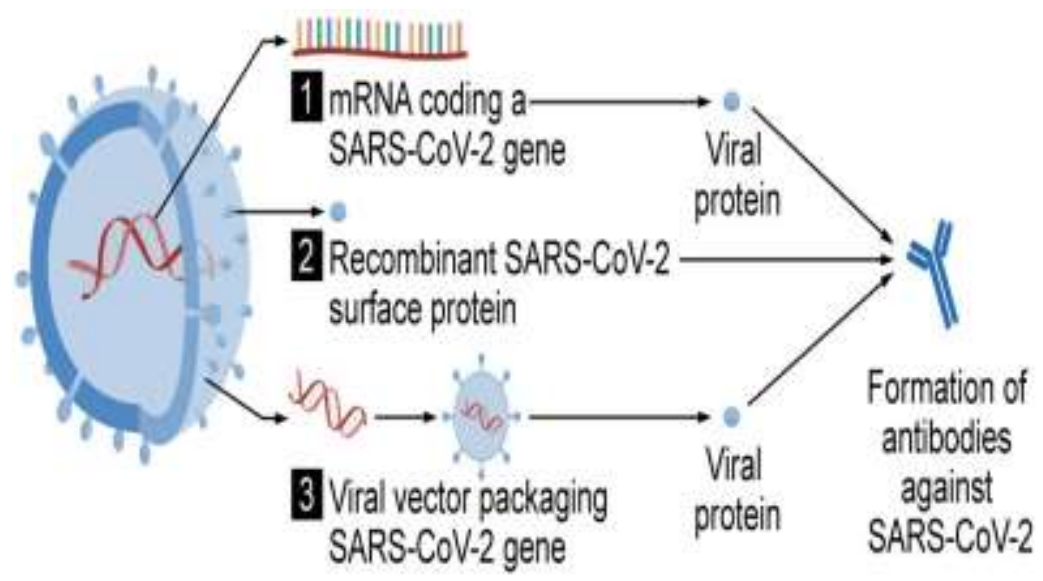

Source: GAO. | GAO-20-583SP

Figure (4). Vaccine candidates use different mechanisms, to prompt the body to produce antibodies against SARS-CoV-2

2. Bioengineered vaccines.

\section{1. m-RNA vaccines}

The first candidate, established by National Institute of Allergy and Infectious Diseases scientists and their collaborators, uses a molecule called mRNA specifically coded to generate proteins that will induce an immune response. This is a newer method of vaccine development that has shown promise in animals during the preclinical phase.

2.1.1.The BioNTech technology for the BNT162b2 vaccine candidate is based on use of messenger RNA (mRNA) which 


\section{Doi:10.21608/asajs.2021.143517 Mohamed Ashraf Mohamed}

encodes part of the spike protein found on the surface of the SARS-CoV-2 coronavirus (COVID-19), triggering an immune response against infection by the virus protein. The mRNA is encapsulated in lipid nanoparticles. The mutated version of the spike protein contains two proline substitutions (designated "2P") that cause it to adopt a shape that stimulates neutralizing antibodies ${ }^{[6][7] .}$ In December 2020, this vaccine was administered in USA and approved to be used in united Kingsom 2.1.2. Moderna' m-RNA-1273 is a synthetic messenger RNA strand which encodes the prefusion-stabilized viral spike protein. When administered I/M, it produce antiviral immunity specifically to SARS-CoV-2 Spike protein inside muscle cells. Which reaches peak levels for 24 to 48 hours and can last for a few more days.

Besides, unlike conventional vaccines, which are either made from inactivated pathogen or small subunits of live pathogen, synthesis of the lipid nanoparticle-encapsulated mRNA vaccine does not require the virus. Therefore, it is relatively safe and ready to be tested. If mRNA-1273 proves to be safe for humans and pass the phase I trial, successive evaluation of its efficacy will be carried out immediately. ${ }^{[8]}$

\subsection{DNA vaccine}

2.2.1. INO-4800 is a DNA vaccine candidate created by Inovio Pharmaceuticals. It is also a genetic vaccine that can be brought to human cells and translated into proteins to produce immune responses. Compared to traditional vaccines, genetic vaccines require lower costs of production and easier way of purification. The simple structure of nucleic acids also obviates the risk of incorrect folding, which could occur in recombinant proteinbased vaccines. However, the amount of plasmid delivered and the adequate interval and route of administration are the factors that may influence the immunogenicity of genetic vaccines [9][10].

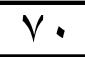




\subsubsection{Covigenix Vaccine}

Unlike traditional vaccines, a DNA-based vaccine involves the direct introduction of a plasmid encoding the antigen(s) against which an immune response is sought and relies on the production of the target antigen in the patient's own cells. This approach offers a number of potential advantages over traditional approaches, including the stimulation of B- and T-cell responses, ease of large-scale manufacture, improved vaccine stability, and the absence of any infectious agent. Until recently, medicines capable of effectively delivering DNA have faced significant challenges in their development. Covigenix Vaccine, Entos' Fusogenix Platform, is a proteo-lipid vehicle (PLV) that uses a novel fusion mechanism to deliver its genetic payload directly inside cells. Entos has developed unique formulations to effectively deliver a wide range of genetic therapies, including plasmid DNA. The use of plasmid DNA in a vaccine will allow Entos to design an optimized payload encoding multiple protein epitopes from key immunogenic SARS-COV-2 proteins. These protein epitopes will stimulate the body's natural antibody production and protective immune response to prevent COVID19 disease ${ }^{[11] .}$

\subsection{Viral vector vaccines}

\subsubsection{Viral replicating vector vaccines}

This approach is based on using a virus that is non-pathogenic or of little danger to humans. In the case of the 12 vaccine candidates of this type currently being studied in humans, the viral vectors are mostly adenoviruses. They have a large group of viruses which usually cause colds and conjunctivitis, among other manifestations.

The candidate uses a virus-adenovirus 26, or Ad26-but researchers have removed its infectious aspects, making it safe as a "vector" to deliver a piece of SARS-CoV-2 to trigger a 
protective immune response. This method is also in clinical trials against HIV and Ebola. The process for developing a new vaccine as outlined by the Food and Drug Administration (FDA) is well established. In the exploratory phase, the target and candidate vaccine are identified. In the preclinical phase, researchers use cells and animals to assess safety and produce evidence of clinical promise, evaluated by the candidate's ability to elicit a protective immune response.

\subsubsection{The Sputnik V vaccine}

It made by the Gamaleya Center for Epidemiology and Microbiology in Moscow, uses adenovirus (Ad) "vectors" to deliver a gene that codes for the surface protein, spike, of SARSCoV-2, the virus that causes COVID-19. The two-dose scheme begins with an Ad26-spike vaccine and is followed by a booster shot 21 days later that contains Ad5 spike. Gamaleya chose two different adenoviruses because of concerns that immune responses to the same vector could lower the impact of the booster shot. Joining the flood of press releases announcing positive results from COVID-19 vaccine trials, developers of Russia's Sputnik V vaccine today reported $91.4 \%$ efficacy from a second interim analysis of more than 18,000 people, bolstering a claim the team made on 11 November 2020 with scant evidence. Whereas the initial report rested on a mere 20 cases of COVID-19, with no details on how they were split between vaccinated and placebo groups, the new analysis is based on 39 cases total, eight among the vaccinated group versus 31 in the much smaller placebo arm. "This is great news not just for Russia, but the world," Kirill Dmitriev, CEO of the Russian Direct Investment Fund that is bankrolling the development of the candidate One adenovirus benefit vaccines is stored in standard refrigerators, rather than needing freezers ${ }^{[12]}$. 
2.3.1.2.Gam-COVID-Vac is a viral two-vector vaccine based on the human adenovirus - a common cold virus - fused with the spike protein of SARS-CoV-2 to stimulate an immune response. The Gam-COVID-Vac vaccine candidate was developed by a government organization that worked on previous coronavirus vaccine candidates. The recombinant adenovirus type-5 (Ad5) and adenovirus type26 (Ad26) are both used as vectors in the vaccine. The Ad26 based vaccine is used on the first day and the Ad5 vaccine is used on the 21st day to boost response. The liquid form of the vaccine must be stored at $-18{ }^{\circ} \mathrm{C}\left(0{ }^{\circ} \mathrm{F}\right)$, and a freeze-dried form is currently being tested that would allow storage at the standard temperature of $2-8{ }^{\circ} \mathrm{C}\left(36-46^{\circ} \mathrm{F}\right)^{[13]}$.

\subsubsection{Non-replicating viral vector vaccines}

\subsubsection{The ChAdOx1 nCoV-19 vaccine (AZD1222)} AstraZeneca's vaccine

It consists of the replication-deficient simian adenovirus vector ChAdOx1, containing the full-length structural surface glycoprotein (spike protein) of SARS-CoV-2, with a tissue plasminogen activator leader sequence. ChAdOx1 nCoV-19 expresses a codon-optimized coding sequence for the spike protein (GenBank accession number MN908947).This vaccine, developed by the University of Oxford, has entered a phase I/II clinical trial (NCT04324606). The non-replicating nature of adenovirus in the host makes it relatively safe in children and individuals with underlying diseases. Besides, the adenovirusbased vectors are characterized by a broad range of tissue tropism that covers both respiratory and gastrointestinal epithelium, the two main sites that express the ACE-2 receptor of SARS-CoV-2. However, the possibility of dominant immunogenicity toward the vector genes rather than the transgenes should always be considered ${ }^{\text {[14] }}$. 


\section{Doi:10.21608/asajs.2021.143517 Mohamed Ashraf Mohamed}

\subsection{Recombinant proteins}

The second candidate uses a recombinant protein, which is produced by genetically engineering bacteria or other cells to produce a protein that mimics part of the spike protein found on the surface of the SARS-CoV-2 virus. The spike protein alone does not cause an infection but may be sufficient to produce an immune response. Recombinant protein vaccines are already being used successfully against other viruses, such as the human papillomavirus (HPV), which can cause cervical cancer. Recombinant protein vaccines fall into two categories: subunit and virus-like particle vaccines. For subunit protein vaccines, a viral protein is produced in large quantities in a living "factory," such as a bacterium, plant, mammalian or insect cell. When the viral protein is presented to the immune system, it triggers a reaction.

\subsubsection{Subunit vaccines}

The University of Queensland (QLD, Australia) is leveraging on its $\mathrm{S}$-spike vaccine. The candidate has been developed via molecular clamp technology, which uses a lab-created polypeptide to pin the spike protein in its tortile position so that the body's immune system can target it before the virus has a chance to activate. Stabilized Subunit Vaccines Enveloped viruses require fusion of the viral membrane with the host cell membrane for infection. This process involves the conformational change of the viral glycoprotein from the prefusion form to the post-fusion form. Although the pre-fusion glycoproteins are relatively unstable, they are still able to elicit strong immune responses. Thus, the University of Queensland is developing a stabilized subunit vaccine based on the molecular clamp technology, which would allow recombinant viral proteins to stably remain in their pre-fusion form. Previously applied to influenza virus and Ebola virus, molecular clamp vaccines have proved their capacity to induce the production of neutralizing 
antibodies. They were also reported to be potent after two weeks at $37{ }^{\circ} \mathrm{C}$. Subunit vaccines have highest degree of safety profile however, have low immunogenicity and requires multiple booster doses to induce adequate immunity. On the other hand, subunit vaccines are also limited in their ability to induce cell mediated immune response ${ }^{[15]}$.

\subsubsection{Virus-like particle vaccines}

Virus-like particle vaccines are composed of a set of viral proteins that mimic the shape of the virus. This particle "pseudovirus" is an empty shell, devoid of genetic material and noninfectious, but this does not prevent the immune system from recognizing it. The 13 subunit vaccine candidates currently in Phase 1, 2 or 3 clinical trials are composed of either the entire spike protein or a specific portion of the spike protein called the 'receptor binding domain'.

\subsection{Nano particle-Based Vaccines}

Nanoparticle-based platforms represent an alternative strategy to incorporate antigens. Through encapsulation or covalent functionalization, nanoparticles can be conjugated with antigenic epitopes, mimic viruses and provoke antigen-specific lymphocyte proliferation as well as cytokine production. In addition, mucosal vaccination through intranasal or oral spray can not only stimulate immune reactions at the mucosal surface, but also provoke systemic responses. This demonstrates the potential of nanoparticle-based vaccines to protect humans against respiratory viruses that cause systemic symptoms. Nano vaccines Evoke a strong immune response ,as shown in figure (5), with advantage of nano-sized range, high antigen load, accelerate immunogenicity, controlled antigen presentation, more retention in lymph node, reduce vaccine dose ${ }^{[16]}$. 


\section{Doi:10.21608/asajs.2021.143517 Mohamed Ashraf Mohamed}

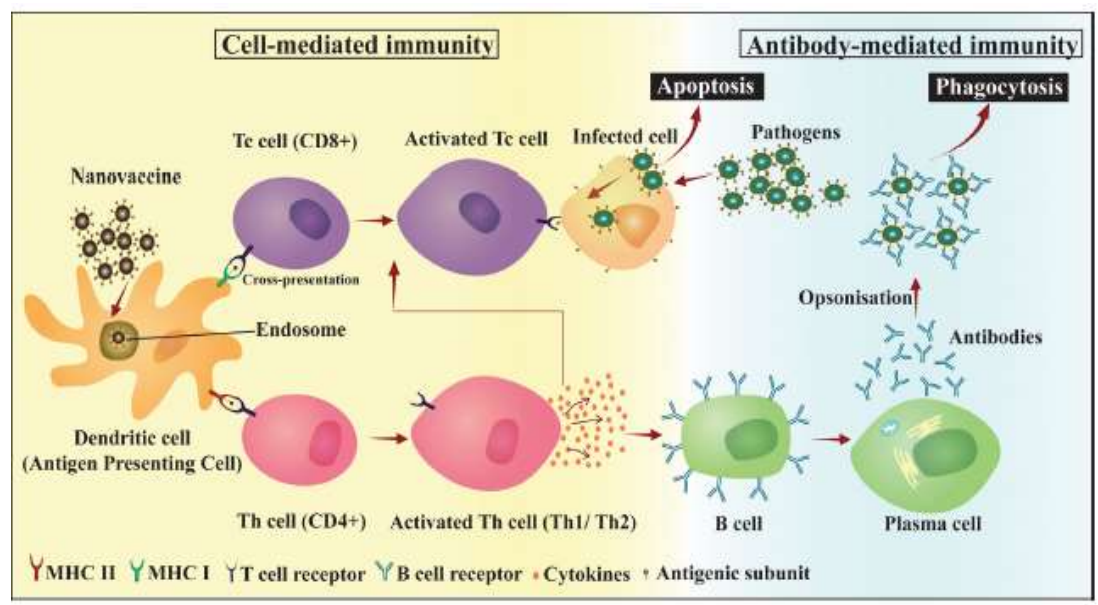

Figure (5): Activation of adaptive immunity by nanoVaccines: uptake and presentation of APCs produce cell and antibody mediated immune responses leading to Apoptosis of infected cell and phagocytosis of antibody-pathogen complex

\subsubsection{NVX-CoV2373}

It is a protein subunit vaccine that contains the spike protein of the SARS-CoV-2 molecule. In January 2020, Novavax announced development of a vaccine candidate, named NVXCoV2373, to establish immunity to SARS-CoV-2.Novavax; Inc. is producing a nanoparticle-based vaccine using antigens derived from the coronavirus $S$ protein. The protein is stably expressed in the baculovirus system, and the product is anticipated to enter phase I trial summer 2020.Novavax entered the final stages of testing its coronavirus vaccine in the UK. Another large trial was announced to start by October 2020 in the US ${ }^{[17]}$.

\section{Fast approval and license of Covid 19 vaccines}

During clinical trials, more human subjects are added at each successive phase. Safety, efficacy, proposed doses, schedule of immunizations, and method of delivery are evaluated. The next phase is FDA approval and licensure, which includes oversight of manufacturing and post market surveillance, and may include

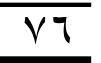


Phase IV trials to monitor safety and efficacy, potency, purity, and other potential uses. At any phase, the process can be terminated for various reasons including detection of adverse events, such as serious side effects. FDA has four programs to facilitate and expedite the review and approval of new therapies for the treatment and prevention of serious or life threatening conditions, such as COVID-19. Fast Track, Breakthrough Therapy, Accelerated Approval, and Priority Review allow for expedited processes, such as overlapping vaccine development phases, to bring vaccines to market more quickly. Vaccine developers could potentially use any or all of these programs for vaccine candidates in the United States. FDA can also issue Emergency Use Authorizations (EUA) for review of vaccine candidates that have not completed all phases of development if there is sufficient scientific evidence on the product's safety, effectiveness, risks, and benefits ${ }^{[18]}$. 


\section{Doi:10.21608/asajs.2021.143517 Mohamed Ashraf Mohamed}

\section{References}

1.Kumar, Sudeep, Raju Sunagar, and Edmund J. Gosselin (2019).“'Bacterial Protein Toll-Like-Receptor Agonists: A Novel Perspective on Vaccine Adjuvants." Frontiers in immunology 10 (2019): 1144.

2. GAO (U.S.GOVERNMENT ACCOUNTABILITY OFFICE). Analysis of GAO-20-215SP, FDA, HHS, and pharmaceutical research and manufactures of America (PhRMA)documentation, GAO20-583SP, SCIENCE \& TECH SPOTLIGHT:

COVID-19 Vaccine Development, Published: May 26, 2020.

3. Candanosa, R.M. (2020) .Here's how nanoparticles could help us get closer to a treatment for COVID-19.

https://news.northeastern.edu/2020/03/04/heres-how-

nanoparticles-could-help-us-get-closer-to-a-treatment-for-covid-

19/

(Accessed:

07

September2020).https://www.labnews.co.uk/article/2030847/fast tracking nano vaccines-to-fight-coronavirus.

4. Xia S, Duan K, Zhang Y, et al. (2020). Effect of an inactivated vaccine against SARS-CoV-2 on safety and immunogenicity outcomes: interim analysis of 2 randomized clinical trials. JAMA 2020; 324: 1-10.

5.Xia S, Zhang Y, Wang Y, et al. (2020).Safety and immunogenicity of an inactivated SARS-CoV-2 vaccine, BBIBP-CorV: a randomized, double-blind, placebo-controlled, phase 1/2 trial. Lancet Infect Dis 2020; published online Oct 15. https://doi.org/10.1016/S1473-3099(20)30831

6. Walsh, EE; Frenck, RW ,et.al. (2020). "Safety and immunogenicity of two RNA-based Covid-19 vaccine candidates". The New England Journal of Medicine: NEJMoa2027906.doi:10.1056/NEJMoa2027906. PMC 7583697. PMID 33053279.

7. Clinical Trials (2020) Pfizer and BioNTech report positive data of second Covid-19 vaccine [online]. 
Availableat:https://www.clinicaltrialsarena.com/news/pfizerbiontech-second-vaccine-2/ (Accessed: 06 September 2020)

8. Clinical trial government (2020). History of Changes for Study: (NCT04283461).Safety and Immunogenicity Study of 2019-nCoV Vaccine (mRNA-1273) for Prophylaxis of SARSCoV-2 Infection (COVID-

19).https://clinicaltrials.gov/ct2/history/NCT04283461.

9. Sheahan, T.P.; Sims, A.C.; Leist, S.R.; Schäfer, A.; Won, J.; Brown, A.J.; Montgomery, S.A.; Hogg, A.; Babusis, D.; Clarke, M.O.; et al.(2020) Comparative therapeutic efficacy of remdesivir and combination lopinavir, ritonavir, and interferon beta against MERS-CoV. Nat. Commun. 2020, 11, 1-14.

10. Lee, J.-W.; Gupta, N.; Serikov, V.; Matthay, M.A.(2009) Potential application of mesenchymal stem cells in acute lung injury. Expert Opin. Boil. 2009, 9, 1259-127.

11. https://www.standard-freeholder.com/pmn/press-releases$\mathrm{pmn} /$ business-wire-news-releases-pmn/entos-pharmaceuticals-isdeveloping-a-dna-vaccine-against-covid-19-using-its-fusogenixnanomedicine-platform/wcm/eb64d2bd-7442-49bf-939ca50afba7b2c9(2020).

\section{Kai Kupferschmidt (2020)}

https://www.sciencemag.org/news/2020/11/more-data-its-covid19-vaccine-russia-institute-offers-new-evidence-success, Nov. 24, 2020

13."An Open Study of the Safety, Tolerability and Immunogenicity of the Drug 'Gam-COVID-Vac' Vaccine against COVID-19". ClinicalTrials.gov. 22 June 2020.

14. Agostini, M.L.; Andres, E.L.; Sims, A.C.; Graham, R.L.; Sheahan, T.P.; Lu, X.; Smith, E.C.; Case, J.B.; Feng, J.Y.; Jordan, R.; et al.(2018) Coronavirus Susceptibility to the Antiviral Remdesivir (GS-5734) Is Mediated by the Viral 
Polymerase and the Proofreading Exoribonuclease. mBio 2018, 9.

15. Chappell, K.; Watterson, D.; Young, P.(2018). Rapid response pipeline for stabilized subunit vaccines. In Proceedings of the Vaccine Technology VII, Mont Tremblant, QC, Canada, 17-22 June 2018.

16. Bhardwaj, P. and Bhatia, E., et.al, (2020).Advancements in prophylactic and therapeutic nano vaccines. Acta Biomaterilia,108,1-21,2020.

17. Sara Gilgore (2020). "Novavax is working to advance a potential coronavirus vaccine. So are competitors". Washington Business, (February 26, 2020). Journal. Archived from the original on March 16, 2020. Retrieved March 6, 2020.

18.Le, Tung T., Zacharias Andreadakis, Arun Kumar, Raúl G. Román, Stig Tollefsen, Melanie Saville, and Stephen Mayhew (2020). "The COVID-19 vaccine development landscape." Nature Reviews Drug Discovery, vol. 19 (2020): pp. 305-306. 